\title{
Video Article \\ Invasion of Human Cells by a Bacterial Pathogen
}

\author{
Andrew M. Edwards ${ }^{1}$, Ruth C. Massey ${ }^{1}$ \\ ${ }^{1}$ Department of Biology and Biochemistry, University of Bath
}

Correspondence to: Ruth C. Massey at r.c.massey@bath.ac.uk

URL: https://www.jove.com/video/2693

DOI: doi:10.3791/2693

Keywords: Infection, Issue 49, Bacterial pathogen, host cell invasion, Staphylococcus aureus, invasin

Date Published: 3/21/2011

Citation: Edwards, A.M., Massey, R.C. Invasion of Human Cells by a Bacterial Pathogen. J. Vis. Exp. (49), e2693, doi:10.3791/2693 (2011).

\section{Abstract}

Here we will describe how we study the invasion of human endothelial cells by bacterial pathogen Staphylococcus aureus. The general protocol can be applied to the study of cell invasion by virtually any culturable bacterium. The stages at which specific aspects of invasion can be studied, such as the role of actin rearrangement or caveolae, will be highlighted. Host cells are grown in flasks and when ready for use are seeded into 24-well plates containing Thermanox coverslips. Using coverslips allows subsequent removal of the cells from the wells to reduce interference from serum proteins deposited onto the sides of the wells (to which $S$. aureus would attach). Bacteria are grown to the required density and washed to remove any secreted proteins (e.g. toxins). Coverslips with confluent layers of endothelial cells are transferred to new 24 -well plates containing fresh culture medium before the addition of bacteria. Bacteria and cells are then incubated together for the required amount of time in $5 \% \mathrm{CO}_{2}$ at $37^{\circ} \mathrm{C}$. For $\mathrm{S}$. aureus this is typically between 15-90 minutes. Thermanox coverslips are removed from each well and dip-washed in PBS to remove unattached bacteria. If total associated bacteria (adherent and internalised) are to be quantified, coverslips are then placed in a fresh well containing $0.5 \%$ Triton X-100 in PBS. Gentle pipetting leads to complete cell lysis and bacteria are enumerated by serial dilution and plating onto agar. If the number of bacteria that have invaded the cells is needed, coverslips are added to wells containing $500 \mu$ lissue culture medium supplemented with gentamicin and incubation continued for $1 \mathrm{~h}$, which will kill all external bacteria. Coverslips can then be washed, cells lysed and bacteria enumerated by plating onto agar as described above. If the experiment requires direct visualisation, coverslips can be fixed and stained for light, fluorescence or confocal microscopy or prepared for electron microscopy.

\section{Video Link}

The video component of this article can be found at https://www.jove.com/video/2693/

\section{Protocol}

The following protocol will describe the study of endothelial cell invasion by $S$. aureus but can theoretically be used to study cellular invasion by any culturable bacterium. Stages specific to $S$. aureus and endothelial cells are indicated.

\section{Preparation of Bacteria}

1. Culture $\mathrm{S}$. aureus strains for $4-16 \mathrm{~h}$ (depending on growth phase required) in $10 \mathrm{ml}$ Brain-Heart Infusion (BHI) broth at $37^{\circ} \mathrm{C}$ in air with shaking at $200 \mathrm{rpm}$. These growth conditions are specific for $S$. aureus and may need to be adapted for other bacteria.

2. Wash bacteria three times in Dulbecco's Modified Eagle's medium (DMEM; Invitrogen) by alternate rounds of centrifugation at room temperature $(5,000 \times \mathrm{g}, 10 \mathrm{~min})$, removal of the culture supernatant and resuspension of the bacterial pellet in an equivalent volume of DMEM. Measure the optical density of the resulting suspension of bacteria which can then be adjusted as required. For $S$. aureus, we prepare a suspension at $\mathrm{OD}_{600}=1$, which corresponds to $\sim 10^{9} \mathrm{cfu} \mathrm{ml}^{-1}$.

\section{Endothelial Cell Culture}

1. Culture the endothelial cell line EA.hy $926{ }^{1}$ in DMEM supplemented with foetal bovine serum (FBS; $\left.10 \%\right)$ and L-glutamine $(2 \mathrm{mM})$ at $37^{\circ} \mathrm{C}$ in $5 \% \mathrm{CO}_{2}$. Alternatively, pooled primary human umbilical vein endothelial cells (HUVECs) can be purchased from Lonza (Basel, Switzerland) and cultured in endothelial basal medium supplemented with $2 \%$ FBS, bovine brain extract (including heparin), human endothelial growth factor and hydrocortisone at $37^{\circ} \mathrm{C}$ in $5 \% \mathrm{CO}_{2}$ according to manufacturer's instructions (Lonza). These growth conditions are specific for these cells and may need to be adapted for other host cell types.

2. Grow endothelial cells in T75 flasks to complete confluency, verified by eye.

3. Prepare the 24-well plates for the insertion of the coverslips. Fine forceps (flame sterilised) are needed to move the coverslips, which have one opaque and one shiny surface. Place coverslips in the wells of the 24-well plate with the opaque surface facing upwards to allow cell attachment.

4. Liberate cells from the T75 flask with $3 \mathrm{ml}$ trypsin-EDTA $(0.25 \%)$ and add to $10 \mathrm{ml}$ of the relevant culture medium.

5. Add $500 \mu \mathrm{l}$ of resuspended cells to 24 -well plates containing Thermanox glass coverslips. One T75 confluent flask of cells provided sufficient cells for two 24-well plates, resulting in approximately $5 \times 10^{5}$ cells per well (in $500 \mu$ l medium). 
6. Incubate plates for $48 \mathrm{~h}$ as described above, and verify $100 \%$ cell confluency by inverted light microscopy.

7. Dip-wash coverslips in PBS and add to new 24-well plates containing $490 \mu \mathrm{l}$ DMEM containing $10 \%$ FBS in each well.

8. To examine the role of specific metabolic processes in cell invasion, inhibitors can be added to the cultured cells $1 \mathrm{~h}$ prior to the addition of bacteria and concentrations maintained during the assay. For example, to determine the role of actin rearrangement in $S$. aureus invasion of endothelial cells, $50 \mu \mathrm{M}$ cytochalasin $\mathrm{D}$ can be added; or for the role of caveolae, $5 \mathrm{mM}$ methyl- $\beta$-cyclodextrin can be added.

\section{Invasion Assay}

1. Add $10 \mu \mathrm{l}$ of washed bacteria (resulting in approximately $2 \times 10^{7} \mathrm{cfu} \mathrm{ml}^{-1} \mathrm{~S}$. aureus ) to each well containing a washed coverslip with a confluent layer of endothelial cells in $490 \mu$ l DMEM containing $10 \%$ FBS.

2. Incubate for $15-90$ minutes at $37^{\circ} \mathrm{C}$ in $5 \% \mathrm{CO}_{2}$.

3. To measure the total number of bacteria associated with the cells (adherent and internalised), dip-wash the coverslips three times in PBS and add to fresh wells containing $500 \mu \mathrm{l} 0.5 \%$ Triton X-100 in PBS. To ensure the cells fully lyse and release all the internalised bacteria, pipette the several times, pointing the tip of the pipette directly at the surface of the coverslip.

4. Enumerate the bacteria by plating the liquid suspension (or dilutions of this where necessary) onto the surface of TSA plates. Since TX-100 will lyse many Gram-negative bacteria, saponin can be used instead ${ }^{2}$.

5. To measure the number of internalised bacteria, remove the culture supernatant from each well containing unbound bacteria and replace with $500 \mu \mathrm{l}$ DMEM/10\% FBS supplemented with $200 \mathrm{\mu g} \mathrm{ml}^{-1}$ gentamicin. We routinely use gentamicin rather than lysostaphin here as it is cheaper and allows us to switch between experiments using different types of bacteria (e.g. Staphylococci and Lactococci) without having to change the experimental protocol.

6. Incubate plates at $37^{\circ} \mathrm{C}$ in $5 \% \mathrm{CO}_{2}$ for 60 min to kill all extracellular bacteria.

7. Wash coverslips 3 times in PBS, lyse and enumerate by plating onto TSA as described for the adhesion assay above.

8. In some cases it may be preferable to visually count the number of bacteria using light microscopy. In this instance, and specific to $S$. aureus cell invasion, use lysostaphin $\left(10 \mu \mathrm{g} \mathrm{ml}^{-1}\right)$ instead of gentamicin to physically destroy the extracellular bacteria.

9. Incubate coverslips for $20 \mathrm{~min}$ at $37^{\circ} \mathrm{C}$ in $\mathrm{CO}_{2}$ in the lysostaphin solution, then rinse and fix with Cytopath (Cellpath).

10. Flood the coverslips with crystal violet $(0.5 \%$, w/v) for $5 \mathrm{~min}$.

11. Dip-rinse in water, air-dry and mount onto glass slides. The number of bacteria per $\mathrm{mm}^{2}$ of confluent endothelial cells can be quantified using light microscopy.

\section{Representative Results:}

Expression of fibronectin binding proteins (FnBPA and FnBPB) on the surface of $S$. aureus confers the ability to invade endothelial cells. Recent work has redefined the fibronectin-binding domain of FnBPA ${ }^{3}$. Wild-type (WT) S. aureus 8325.4 invades endothelial cells with high efficiency, whilst a strain lacking both FnBPA and $\mathrm{B}(\Delta f n b)$ showed significantly reduced levels of internalisation (Figure $1 \mathrm{~A})$. Complementation of the mutant with a plasmid encodingfnb A minus the fibronectin-binding domain ( $p F n R 0)$ did not promote invasion (Figure 1A). By contrast, complementation of the mutant with a plasmid encoding the entire fnb A gene ( $p F n B A 4)$ restored invasion to WT levels (Figure 1A).

The role of FnBPA in endothelial cell invasion can also be demonstrated using the heterologous expression host Lactococcus lactis . Expression of plasmid-encoded FnBPA in L. lactis (pRM9 ${ }^{9}$ ) did not significantly enhance adhesion to endothelial cells compared to bacteria expressing no FnBPA (CTL) (open bars, Figure 1B). By contrast, FnBPA-expressing L. lactis invaded endothelial cells at significantly higher levels than the nonexpressing strain (closed bars, Figure 1B).

Experiments were performed four times in duplicate and the mean \pm standard deviation is presented. * represent data that are statistically significantly different $(p=<0.05)$ from WT or CTL values.
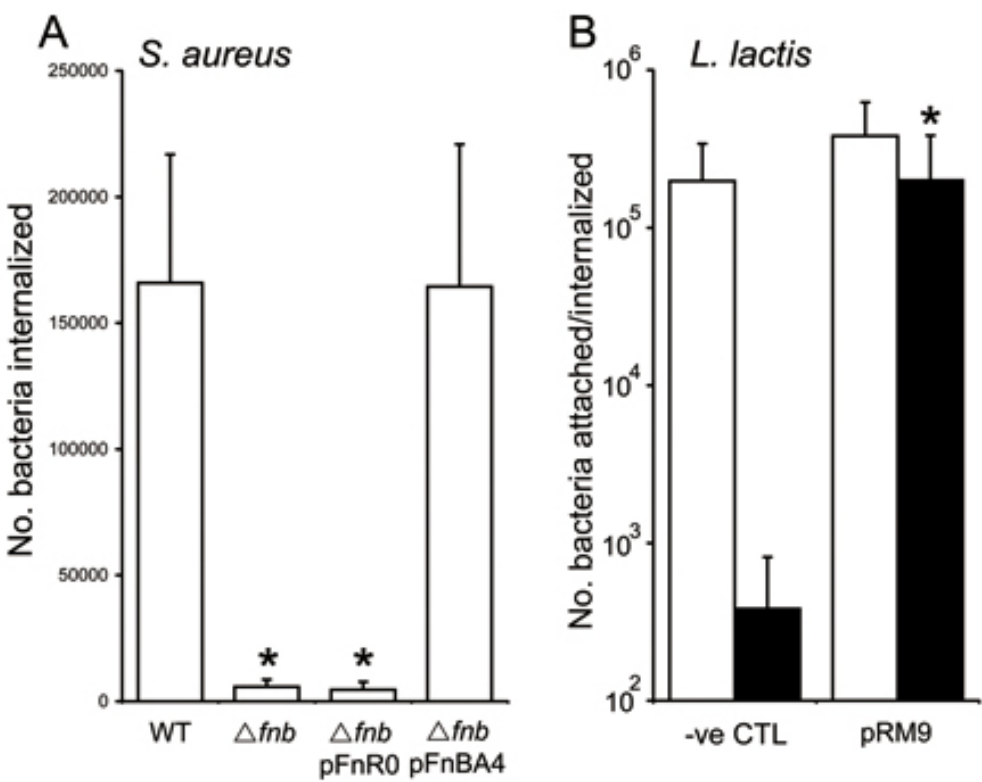
Figure 1. Invasion of EA. Hy926 endothelial cells by S. aureus (A) or L. lactis (B).

\section{Discussion}

The assay we describe is based on the gentamicin protection assay, which has been used widely to study the invasion of host cells by bacteria. Observations of the inability of gentamicin and other antibiotics to kill intracellular bacteria were utilised in early studies on the invasion of host cells by bacteria ${ }^{4-8}$. The use of 24,48 or even 96 -well plates allows the generation of large amounts of quantitative, reproducible data in a short period of time and at relatively low cost. The assay is also attractive because it requires no specialist equipment, it can be tailored to work with various bacteria and cells, and can be used to study the role of both bacterial and host cell processes in invasion ${ }^{9}$. Indeed, since the earliest experiments, the gentamicin protection assay has been widely employed to measure host cell invasion by a range of different bacteria or even combinations of bacteria ${ }^{10,11}$. Whilst the core principles are the same, many subtle variations in the method have been reported. In this article we have described our version and indicated where modifications may be necessary for other bacteria or host cells. When designing an experiment to measure host cell invasion using this approach there are a number of important points to consider:

Bacterial sensitivity to gentamicin. This may seem obvious, but it is important to ensure that the bacterium being studied is susceptible to gentamicin at the concentration, temperature and over the time duration to be used. In the case of insensitivity, it may be possible to use other antibiotics $^{5}$. An alternative approach can be to use lytic enzymes (lysostaphin, mutanolysin, lysozyme) ${ }^{12}$.

Incubation and inoculum. When performing this assay for the first time it is important to establish the optimal incubation times and bacterial inoculum to be used. Therefore, initial experiments should examine both adhesion and invasion over time ( $5 \mathrm{~min}$ to up to $6 \mathrm{~h}$ ). It is also important to assess how invasion is affected by the multiplicity of infection (MOI; number of bacteria per cell). In general it is best to use the fewest number of bacteria possible as this will reduce damage to the cultured cells. Important differences between strains may be missed if extended incubation periods or large inocula are used ${ }^{9,13}$.

Cellular damage. It is important to wash bacteria prior to use. However, cellular damage is not restricted to toxin production. Bacterial invasion, induction of apoptosis and disruption of normal cellular functions can all cause cellular damage. This can lead to penetration of gentamicin into the cell, killing internalised bacteria and giving the impression that invasion has not occurred ${ }^{14}$.

Incubation conditions. The temperature and composition of the culture medium can have a significant impact on invasion. For example, invasion of HeLa cells by Streptococcus pyogenes is dependent upon the presence of soluble fibronectin, which is typically present in serum ${ }^{15}$. Another consideration is bacterial growth in the culture medium during the course of the experiment.

Culture and quantification of intracellular bacteria. Although TX-100 may not kill intracellular bacteria, it may inhibit their growth. As such, it is important to check bacterial replication on solid media in the presence of the detergent. Where affected, it may be possible to use lower concentrations of the detergent or to use an alternative such as saponin. A benefit of the gentamicin protection assay over the crystal violet staining and microscopy assay is that it is less labor intensive, and it allows the detection and differentiation of sub-populations of internalised bacteria. For example, S. aureus can produce either a normal colony type (NCT) or the small colony variant (SCV) ${ }^{16}$, which would not be distinguishable by microscopy of individual bacterial cells. A benefit of the crystal violet staining and microscopy assay over the gentamicin protection assay is cell clumping can be accounted for and that intracellular bacteria that enter a 'viable but non-culturable' state will be detected

Examining the role of host cell processes in bacterial invasion. Many studies have employed inhibitors of host cell function such as cytochalasin $\mathrm{D}$, which interferes with actin rearrangement. Such studies can also include antibodies that target cell-surface receptors and siRNA knockdown of specific genes ${ }^{18}$. It is vitally important to ensure that these treatments do not affect the viability or attachment of cultured cells or have toxic effects on bacteria.

\section{Future directions:}

Because this assay is typically performed in multi-well cell culture plates, it is theoretically possible to adapt it to a high-throughput screening operation, which might involve the examination of libraries of potential cell-function inhibitors, anti-infectives or antibiotics designed to target intracellular bacteria. Alternatively, such an approach could be used to screen mutant libraries to identify genes involved in adhesion, invasion or intracellular survival. In some cases it may be desirable to include shear forces (flow) in the model system, for example when modeling the endothelium, to more closely mimic the in vivo environment. Current advances in the design and manufacture of flow cells and microfluidic cell culture systems provide the possibility of employing the gentamicin protection assay in host-pathogen models that incorporate shear forces.

In summary, the protocol described here is based upon similar approaches used over many years. It is widely applicable to many types of cultured cells and bacterial species and can generate large amounts of data quickly and at relatively little cost.

\section{Disclosures}

No conflicts of interest declared.

\section{Acknowledgements}

This work was funded by the Wellcome Trust (WT 0795880). 


\section{References}

1. Edgell, C.J., McDonald, C.C. \& Graham, J.B.Permanent cell line expressing human factor VIII-related antigen established by hybridization. Proc. Natl. Acad. Sci. U. S. A. 80, 3734-3737 (1983).

2. Makino, S., van Putten, J.P. \& Meyer, T.F. Phase variation of the opacity outer membrane protein controls invasion by Neisseria gonorrhoeae into human epithelial cells. EMBO J. 10, 1307-1315 (1991).

3. Schwarz-Linek, U., Werner, J.M., Pickford, A.R., Gurusiddappa, S., Kim, J.H., Pilka, E.S., Briggs, J.A., Gough, T.S., Höök, M., Campbell, I.D. \& Potts, J.R. Pathogenic bacteria attach to human fibronectin through a tandem beta-zipper. Nature. (423), 177-181 (2003).

4. Magoffin,R.L. \& Spink, W.W. The protection of intracellular Brucella against streptomycin alone and in combination with other antibiotics. J. Lab. Clin. Med. 37, 924-930 (1951).

5. Mandell, G.L. Interaction of intraleukocytic bacteria and antibiotics. J. Clin. Invest. 52, 1673-1679, (1973.)

6. Vaudaux, P. \& Waldvogel, F.A. Gentamicin antibacterial activity in the presence of human polymorphonuclear leukocytes. Antimicrob. Agents Chemother. 16, 743-749, (1979).

7. De Melo, M.A. \& Pechere, J.C. Effect of mucin on Campylobacter jejuni association and invasion on HEp-2 cells. Microb. Pathog. 5, 71-76 (1988).

8. Shaw, J.H. \& Falkow, S. Model for invasion of human tissue culture cells by Neisseria gonorrhoeae. Infect. Immun. 56, 1625-1632, (1988).

9. Edwards, A.M., Potts, J.R., Josefsson, E. and Massey, R.C. Staphylococcus aureus Host Cell Invasion and Virulence in Sepsis is Facilitated by the Multiple Repeats within FnBPA. PLoS Pathog. 6(6): e1000964 (2010).

10. Meyer, D.H., Sreenivasan, P.K. \& Fives-Taylor, P.M. Evidence for invasion of a human oral cell line by Actinobacillus actinomycetemcomitans. Infect. Immun. 59, 2719-2726 (1991).

11. Edwards, A.M., Grossman, T.J. \& Rudney, J.D. Fusobacterium nucleatum transports noninvasive Streptococcus cristatus into human epithelial cells. Infect. Immun. 74, 654-662 (2006).

12. Menzies, B.E. \& Kourteva, I. Internalization of Staphylococcus aureus by Endothelial Cells Induces Apoptosis. Infect. Immun.66, 5994-5998 (1998).

13. Schröder, A., Schröder, B., Roppenser, B., Linder, S., Sinha, B., Fässler, R. \& Aepfelbacher, M. Staphylococcus aureus fibronectin binding protein-A induces motile attachment sites and complex actin remodeling in living endothelial cells. Mol. Biol. Cell. 17, 5198-5210 (2006).

14. Molinari, G., Rohde, M., Talay, S.R., Chhatwal, G.S., Beckert, S. \& Podbielski, A. The role played by the group A streptococcal negative regulator Nra on bacterial interactions with epithelial cells. Mol. Microbiol. 40, 99-114 (2001).

15. Cue, D., Dombek, P.E., Lam, H. \& Cleary, P.P. Streptococcus pyogenes serotype M1 encodes multiple pathways for entry into human epithelial cells. Infect. Immun. 66, 4593-4601 (1998).

16. von Eiff, C. Staphylococcus aureus small colony variants: a challenge to microbiologists and clinicians. Int J. Antimicrob. Agents. $31,507-510$ (2008).

17. Oliver, J.D. Recent findings on the viable but nonculturable state in pathogenic bacteria. FEMS Microbiol. Rev. 34, 415-425 (2010).

18. Wang, B., Yurecko, R.S., Dedhar, S. \& Cleary, P.P. Integrin-linked kinase is an essential link between integrins and uptake of bacterial pathogens by epithelial cells. Cell. Microbiol. 8, 257-266 (2006). 